\title{
An Analytic Solution For Hedge Ratios On Stocks With Dividends And The Accuracy Of Black-Scholes Hedge Ratios
}

Kanwal Sachdeva, (E-mail: Kanwal.Sachdeva@sdsu.edu), San Diego State University William Sterk, (E-mail: William.Sterk@sdsu.edu), San Diego State University

\begin{abstract}
The Black-Scholes model continues to be the standard option pricing model discussed in virtually all corporate finance and investments texts and continues to be widely used in practice. The model's associated hedge ratio has also been widely used for hedging purposes. The associated hedge ratio (or delta) is determined as part of calculating the Black-Scholes option value. However, the original model assumes no dividends on the underlying stock. The model has been modified to allow for dividends, but the modification does not lead to values as precise as other models, such as the Roll-Geske-Whaley model that specifically account for dividends. Empirical research has shown that the RGW model values are closer to actual market prices than the modified BlackScholes values. This paper is primarily concerned with the hedge ratio. We derive an analytic solution for a more accurate hedge ratio based on the RGW model. The paper is then concerned with how large the errors are associated with using the BS approximation rather than the more complicated model that specifically accounts for dividends. We find that although there are times when the BS approximation can be accurate, at other times the differences can be significant. These differences are related to the size of the dividend, the difference between the time to expiration and the time to ex-dividend, the rate of interest, the stock volatility, and the degree to which the option is in-the-money.
\end{abstract}

\section{Introduction}

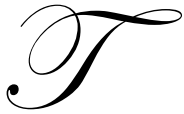

he Black-Scholes hedge ratio (or "delta") developed in conjunction with the Black-Scholes option pricing model (See Black and Scholes (1973) and Black (1975).) is a widely recognized and applied ratio used for hedging purposes. "Delta hedging" schemes to reduce risk are generally discussed in detail in investment and options texts. (See Cox and Rubinstein (1985), Hull (2000), Jarrow and Turnbull (2000), and Figlewski, Silber and Subrahmanyam (1992).) Computational routines and software are now widely available to calculate both BS option values and the associated hedge ratio using either calculators or computers. (See Cox and Rubinstein (1985, pp. 343-345) for a calculator program and Haugen (1997) for computer software.) Unfortunately, the BS model assumes that the underlying stocks do not pay dividends. A modified form of the original BS model has been developed to take dividends into account, however the modification is not exact. Subsequently, Roll (1977), Geske (1979), and Whaley (1981) developed an analytic option pricing formula that specifically accounts for dividends on the underlying stock. The RGW option values have been found by Whaley (1982) and Sterk (1983) to more closely match actual option market prices. We derive a formula for the hedge ratio based on the RGW option valuation model, and compare it to the deltas based on a modified BS model. The paper further explores when the BS approximating ratios can be expected to be significantly different from the theoretically more accurate RGW-based ratio.

Readers with comments or questions are encouraged to contact the authors via email. 


\section{Modified Black-Scholes Hedge Ratios}

The Black-Scholes option-pricing model is well-known as is its associated hedge ratio. The original BS model and associated hedge ratio are presented in equations (1) and (2) respectively.

$$
B S=P N\left(d_{1}\right)-X \exp (-r T) N\left(d_{2}\right)
$$

Where

\begin{tabular}{|l|l|l|}
\hline BS & $=$ & The Black-Scholes option value \\
\hline $\mathrm{P}$ & $=$ & The current stock price \\
\hline $\mathrm{X}$ & $=$ & The exercise price \\
\hline $\mathrm{R}$ & $=$ & The riskless rate of interest \\
\hline $\mathrm{T}$ & $=$ & The time to expiration \\
\hline $\mathrm{N}(\mathrm{a})$ & $=$ & The univariate cumulative normal density function with integral limit a \\
\hline$\sigma$ & $=$ & The instantaneous standard deviation of the stock's rate of return \\
\hline
\end{tabular}

$d_{1}=\frac{\ln (P / X)+\left(r+0.5 \sigma^{2}\right) T}{\sigma \sqrt{T}} \quad, \quad d_{2}=d_{1}-\sigma \sqrt{T}$.

$B S H R=N\left(d_{1}\right)$

Where BSHR is the Black-Scholes hedge ratio.

The original BS model presented above assumes that early exercise is not a possibility and that the underlying stock does not pay dividends. The BS model correctly values American options when the underlying stock does not pay dividends because it will not pay to exercise the option before the maturity date. However, with dividends, it may be beneficial to exercise early if the option is not "dividend-protected" which is the usual case. This is due to the drop in the stock price when it goes ex-dividend. That is, the option may be worth more dead than alive. On the other hand, there are benefits to not exercising at the ex-dividend date. By postponing exercising, the time value of money on the exercise price is saved. In addition, there is an insurance value to holding on to the option. Between the ex-dividend date and the maturity date there may be a large drop in the stock price. If the option is exercised at the ex-dividend date, the option holder now owns the stock instead and suffers the large loss. Alternatively, if exercising is postponed, there would be a smaller dollar loss since the stock price fall will be larger than the call loss in value.

The BS model has been modified to allow for dividend payments and the associated possibility of early exercise. The process calls for computing two BS values -- one assuming no early exercise and a second assuming early exercise. The process is well-summarized by Jarrow and Turnbull (2000, pp. 255-256.) and Sharpe, Alexander, and Bailey (1999, pp. 630-632). The modified BS value, MBS, is the larger of the two values, BS1 or BS2, shown in equations (3) and (4) corresponding to no early exercise or early exercise respectively. The modified BS model has commonly been referred to as the pseudo-American model.

$$
B S 1=S N\left(d_{1}\right)-X \exp (-r T) N\left(d_{2}\right)
$$

Where

$\mathrm{S}=$ Stock price net of escrowed dividend $=\mathrm{P}-\mathrm{D} \exp (-\mathrm{rt})$

$\mathrm{T}=$ time until ex-dividend 
$d_{1}=\frac{\ln (S / X)+\left(r+0.5 \sigma^{2}\right) T}{\sigma \sqrt{T}}, d_{2}=d_{1}-\sigma \sqrt{T}$

and the remaining variables are as defined in (1).

$$
B S 2=S N\left(d_{1}\right)-(X-D) \exp (-r t) N\left(d_{2}\right)
$$

Where

$S=P-D \exp (-r t)$

$d_{1}=\frac{\ln (S /(X-D))+\left(r+0.5 \sigma^{2}\right) t}{\sigma \sqrt{t}} \quad, \quad d_{2}=d_{1}-\sigma \sqrt{t}$

and the remaining variables are as defined in (1).

There are therefore two BS hedge ratios; one associated with the BS model assuming no early exercise, BS1HR, and one assuming early exercise, BS2HR. These are shown in equations (5) and (6) respectively. The modified BS hedge ratio, MBSHR, would then be as shown in equation (5) if BS1 is larger than BS2. If BS2 is larger, the modified BS hedge ratio is given by equation (6).

$B S 1 H R=N\left(d_{1}\right)$

Where $d_{1}$ is defined in (3).

$$
B S 2 H R=N\left(d_{1}\right)
$$

Where $d_{1}$ is as defined in (4).

The problem with the above approach is that the probability of early exercise is generally not equal to 0 or 1 but lies somewhere in between. The further away from 0 or 1 that the actual probability of early exercise is, the less accurate the pseudo-American option and hedge ratio values will be.

\section{The Hedge Ratio Based on the RGW Model}

The RGW option valuation model originally developed by Roll (1977), simplified by Geske (1979), and corrected by Whaley (1981) is shown in equation (7). (The model is also discussed in Jarrow and Rudd (2000, pp. 257-58) and Hull (2000, p. 271)

$$
\begin{aligned}
& R G W=S\left[N_{1}\left(b_{1}\right)+N_{2}\left(a_{1},-b_{1} ;-\sqrt{t / T}\right)\right] \\
& -X \exp (-r T)\left[N_{1}\left(b_{2}\right) \exp (r(T-t))+N_{2}\left(a_{2},-b_{2} ;-\sqrt{t / T}\right)\right] \\
& +\alpha D \exp (-r t) N_{1}\left(b_{2}\right)
\end{aligned}
$$

Where

$\mathrm{RGW}=$ the market value of an American call option 


$$
\begin{array}{ll}
a_{1}=\frac{\ln (S / X)+\left(r+0.5 \sigma^{2}\right) T}{\sigma \sqrt{T}}, & a_{2}=a_{1}-\sigma \sqrt{t} \\
b_{1}=\frac{\ln \left(S / S_{t}^{*}\right)+\left(r+0.5 \sigma^{2}\right) t}{\sigma \sqrt{t}}, & b_{2}=b_{1}-\sigma \sqrt{t}
\end{array}
$$

\begin{tabular}{|l|l|l|}
\hline $\mathrm{N}_{1}(\mathrm{a})$ & $=$ & the univariate cumulative normal density function with integral limit a \\
\hline $\mathrm{N}_{2}(\mathrm{a}, \mathrm{b} ;-\rho)$ & $=$ & $\begin{array}{l}\text { the bivariate cumulative normal density function with upper integral limits a and b and correlation } \\
\text { coefficient }-\rho\end{array}$ \\
\hline$S_{t}^{*}$ & $=$ & the solution to $\mathrm{C}\left(\mathrm{S}_{\mathrm{t}}{ }_{\mathrm{t}}, \mathrm{T}-\mathrm{t}, \mathrm{X}\right)=\mathrm{S}_{\mathrm{t}} \square+\alpha \textrm{D}-\mathrm{X}$, where $\mathrm{C}($.$) is the original \mathrm{BS}$ value \\
\hline $\mathrm{S}$ & $=$ & the current stock price, net of escrowed dividend \\
\hline $\mathrm{T}$ & $=$ & time until expiration \\
\hline $\mathrm{X}$ & $=$ & exercise price \\
\hline $\mathrm{r}$ & $=$ & the riskless rate of interest \\
\hline$\sigma^{2}$ & $=$ & the variance of the rate of return on $\mathrm{S}$ \\
\hline $\mathrm{D}$ & $=$ & the dividend \\
\hline $\mathrm{t}$ & $=$ & the time until the ex-dividend instant \\
\hline$\alpha$ & $=$ & the decline in the stock price at ex-dividend as a proportion of the dividend \\
\hline
\end{tabular}

and the remaining variables are as defined in (1).

This model allows for a probability of early exercise other than only 0 or 1 and is therefore a theoretically more accurate model than the modified BS model. It has also been shown by Sterk (1983) and Whaley (1982) to be more accurate empirically. The hedge ratio associated with the RGW model, RGWHR, derived in Appendix A and shown in equation (8) is also theoretically more accurate than the modified BS hedge ratio.

$$
\begin{aligned}
& R G W H R=N_{1}\left(b_{1}\right)+N_{2}\left(a_{1},-b_{1} ; \rho\right)+\frac{1}{S \sqrt{2 \pi} \sigma}\{ \\
& S\left[\frac{\exp \left[-0.5 b_{1}^{2}\right]}{\sqrt{t}}+\frac{\exp \left[-0.5 a_{1}^{2}\right]}{\sqrt{T}} N_{1}\left(\frac{-a_{1} \rho-b_{1}}{\sqrt{1-\rho^{2}}}\right)-\frac{\exp \left[-0.5 b_{1}^{2}\right]}{\sqrt{t}} N_{1}\left(\frac{a_{1}+b_{1} \rho}{\sqrt{1-\rho^{2}}}\right)\right] \\
& -X \exp [-r T]\left[\frac{\exp \left[-0.5 b_{2}^{2}\right]}{\sqrt{t}} \exp [r(T-t)]+\frac{\exp \left[-0.5 a_{2}^{2}\right]}{\sqrt{T}} N_{1}\left(\frac{-a_{2} \rho-b_{2}}{\sqrt{1-\rho^{2}}}\right)\right. \\
& \left.\left.-\frac{\exp \left[-0.5 b_{2}^{2}\right]}{\sqrt{t}} N_{1}\left(\frac{a_{2}+b_{2} \rho}{\sqrt{1-\rho^{2}}}\right)\right]+\frac{\alpha D \exp [-r t]}{\sqrt{t}} \exp \left[-0.5 b_{2}^{2}\right]\right\} .
\end{aligned}
$$

Differences between the RGW hedge ratio and the modified BS hedge ratios are investigated in the next section.

\section{Differences between the RGW and BS Hedge Ratios}

One would expect the differences between the RGW hedge ratio and the BS hedge ratios to be the largest when the probability of early exercise is further away from 0 or 1 . The probability of early exercise is affected by the size of the dividend, the time remaining between the ex-dividend date and the expiration date, the rate of interest, the volatility of the underlying stock, and the extent to which the option is in-the-money. (See Figlewski et. al. (1990, pp. 142-143).) 


\section{Effect of Dividend Size}

Since the motivation for early exercise is due to dividends on the underlying stock, the larger the dividend, the larger the benefit from exercising early and the probability of doing so. To investigate the effect of the size of the dividend on the difference between the RGW and BS hedge ratios, hedge ratios were computed for various dividends. Results for a stock price of $\$ 40$, exercise price of $\$ 45$, standard deviation of 0.30 , time until ex-dividend of 45 days, time until expiration of 90 days, and risk-free rate of 0.05 are shown in Figure 1.

As can be seen in Panel A of Figure 1, the original BS hedge ratio, BS1HR, which does not allow for early exercise is accurate for a zero dividend as expected. However, as the dividend increases, and with it the probability of early exercise, this approximation becomes very inaccurate. For large dividends the BS ratio assuming early exercise, BS2HR, is more accurate. The modified BS hedge ratio will equal the BS1HR and the RGW ratio for a zero dividend; whereas it equals the BS2HR $=$ RGWHR for very large dividends. The modified BSHR switches from the BS1HR to the BS2HR at a dividend of $\$ 4$ when the call value associated with early exercise becomes larger than the call value associated with no early exercise. For intermediate dividend values, the modified BS hedge ratio will be too small. The error associated with the modified BS hedge ratio approximation is the largest for a dividend of $\$$ 3.5. Here the modified BS hedge ratio will be about 0.1 as compared to a correct RGW value of approximately 0.15. Panel B of Figure 1 shows that for the $\$ 3.50$ dividend, the BS approximation is about $30 \%$ too low. ${ }^{1}$

\section{Figure 1. Effect of Dividend Size on Hedge Ratios}

Panel A shows the hedge ratios. Panel B shows the percentage difference between the modified BlackScholes hedge ratios and the Roll-Geske-Whaley hedge ratios. BS1HR is the hedge ratio assuming no early exercise. BS2HR is the hedge ratio assuming early exercise. MBSHR is the hedge ratio associated with the larger Black-Scholes option value.

Figure 1A

\section{HEDGE RATIOS}

$\mathrm{P}=40 \mathrm{X}=45 \mathrm{t}=.30 \mathrm{t}=45 \mathrm{~T}=90 \mathrm{r}=.05$

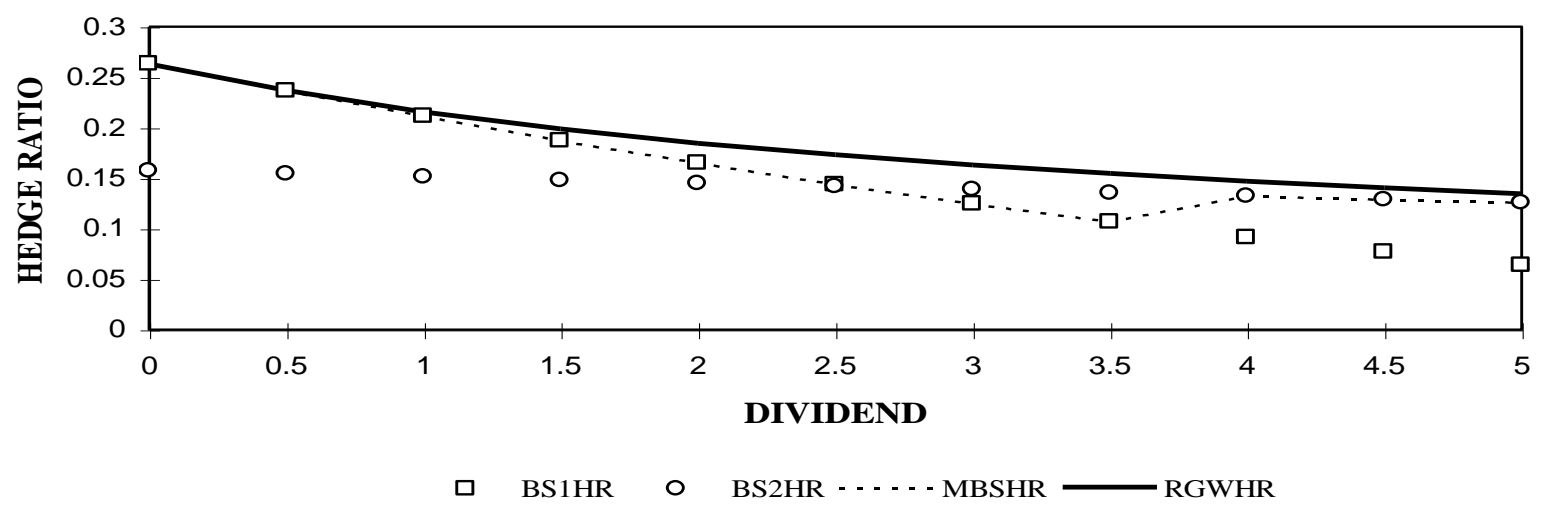

Figure 1B 


\section{$\%$ DIFF HEDGE RATIOS}

$\mathrm{P}=40 \mathrm{X}=45 \quad .30 \mathrm{t}=45 \mathrm{~T}=90 \mathrm{r}=.05$

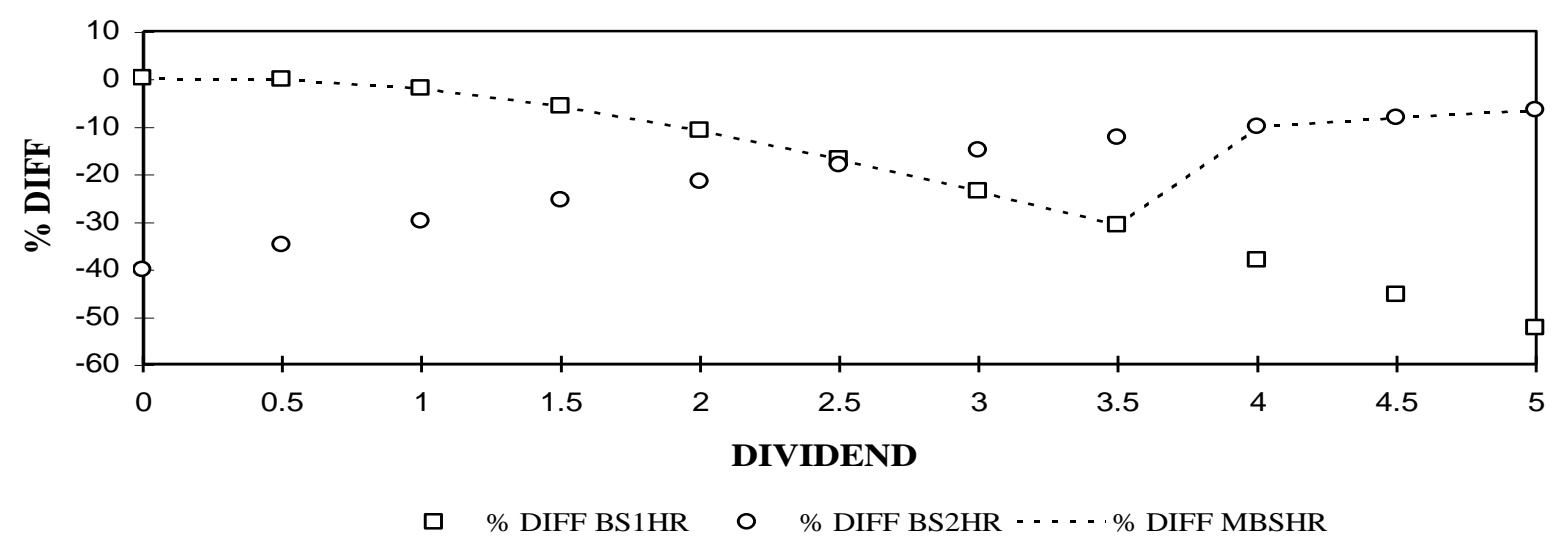

\section{Effect of Difference between $t$ and $T$}

The likelihood of early exercise decreases as the difference between the time to ex-dividend and time to expiration increases. As this period of time increases, there is more of an opportunity cost in terms of the time value of money forgone because the exercise price is paid earlier. Therefore, the BS1HR based on no early exercise should be a good approximation for large t-T, and BS2HR should be a good approximation for small t-T. MBSHR should be close to RGWHR for relatively small and large t-T. This is shown to be the case in Figure 2 where results are shown for the same values of the variables in Figure 1, except the dividend is set at $\$ 1$ and the time until exdividend, $\mathrm{t}$, is allowed to vary.

As can be seen in Figure 2, if the time between ex-dividend and expiration is only five days ( $\mathrm{t}=85$ days), and the BS hedge ratio assuming no early exercise is used, one would be using a hedge ratio which would be about $15 \%$ too low (hedge ratio equals 0.21 instead of 0.25 ). However, BS2HR which assumes early exercise would be accurate (as would also the modified BS hedge ratio). As the difference between $\mathrm{t}$ and $\mathrm{T}$ increases, the original BS hedge ratio becomes more accurate while the BS2HR becomes less accurate. At $\mathrm{t}=45$ days the BS2HR based on early exercise would be $30 \%$ too low. In between, the BS approximation is less accurate. The modified BS approximation error is the largest (approximately $-8 \%$ ) for a difference between the ex-dividend date and expiration date equal to 25 days ( $\mathrm{t}=65$ days ). The MBSHR switches from BS1HR to BS2HR at $\mathrm{t}=75$ days when the BS call value based on early exercise becomes larger than the call value based on no early exercise.

\section{Effect of the Risk-free Rate of Interest}

The opportunity cost associated with exercising the option early (and paying the exercise price sooner) increases as the rate of interest increases. Thus, the likelihood of exercising early should decrease as the rate of interest increases. The BS1HR based on no early exercise should be more accurate at high interest rates, whereas the BS2HR based on early exercise should be more accurate at low interest rates. This is verified in Figure 3 where results are shown for a stock price equal to $\$ 50$, exercise price of $\$ 45$, standard deviation of $30 \%$, time until exdividend of 45 days, time until expiration of 90 days, and dividend of $\$ 1$.

As can be seen in Figure 3, the RGW hedge ratio is not as sensitive to the rate of interest as it is for the other variables. The MBSHR switches from the BS2HR to the BS1HR at an interest rate of 5\%. This is also where

Figure 2. 
Effect of Difference between Time Until Expiration, $T$, and Time Until Ex-Dividend, t, on Hedge Ratios

Panel A shows the hedge ratios. Panel B shows the percentage difference between the modified Black-Scholes hedge ratios and the Roll-Geske-Whaley hedge ratios. BS1HR is the hedge ratio assuming no early exercise. BS2HR is the hedge ratio assuming early exercise. MBSHR is the hedge ratio associated with the larger BlackScholes option value.

Figure 2A

\section{HEDGE RATIOS}

$\mathrm{P}=40 \mathrm{X}=45=.30 \mathrm{D}=1 \mathrm{~T}=90 \mathrm{r}=.05$

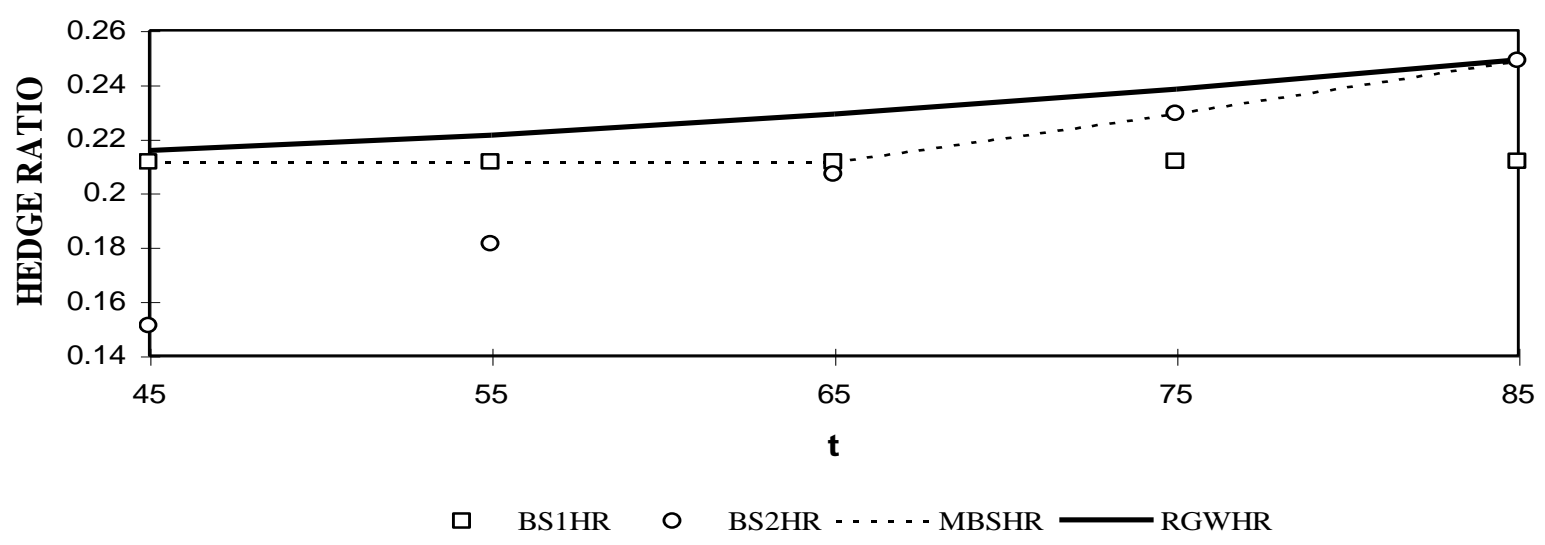

Figure 2B

\% DIFF HEDGE RATIOS

$\mathrm{P}=40 \mathrm{X}=45 \mathrm{C}=.30 \mathrm{D}=1 \mathrm{~T}=90 \mathrm{r}=.05$

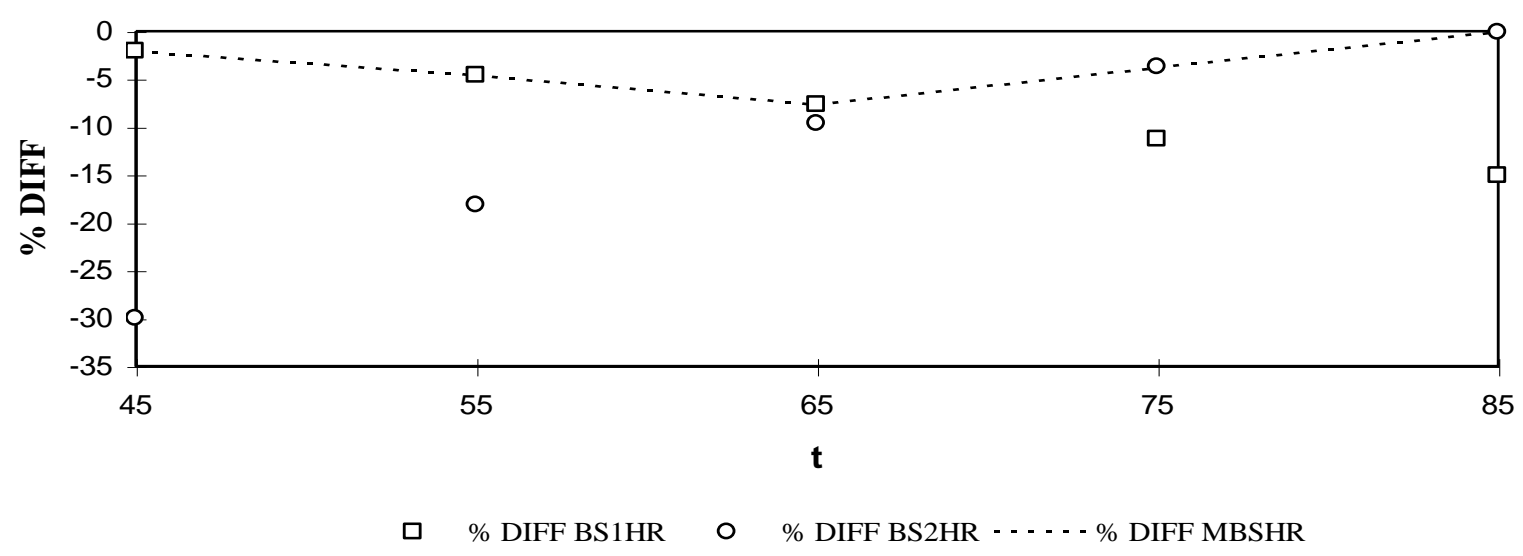


the error associated with using the modified BS approximation is maximized. At this rate, both BS approximations have absolute errors of about $6 \%$.

Figure 3.

\section{Effect of Risk-Free Rate, r, on Hedge Ratios}

Panel A shows the hedge ratios. Panel B shows the percentage difference between the modified Black-Scholes hedge ratios and the Roll-Geske-Whaley hedge ratios. BS1HR is the hedge ratio assuming no early exercise. BS2HR is the hedge ratio assuming early exercise. MBSHR is the hedge ratio associated with the larger Black-Scholes option value.

Figure 3A

HEDGE RATIOS

$\mathrm{P}=50 \mathrm{X}=45 \mathrm{\sigma}=.30 \mathrm{t}=45 \mathrm{~T}=90 \mathrm{D}=1$

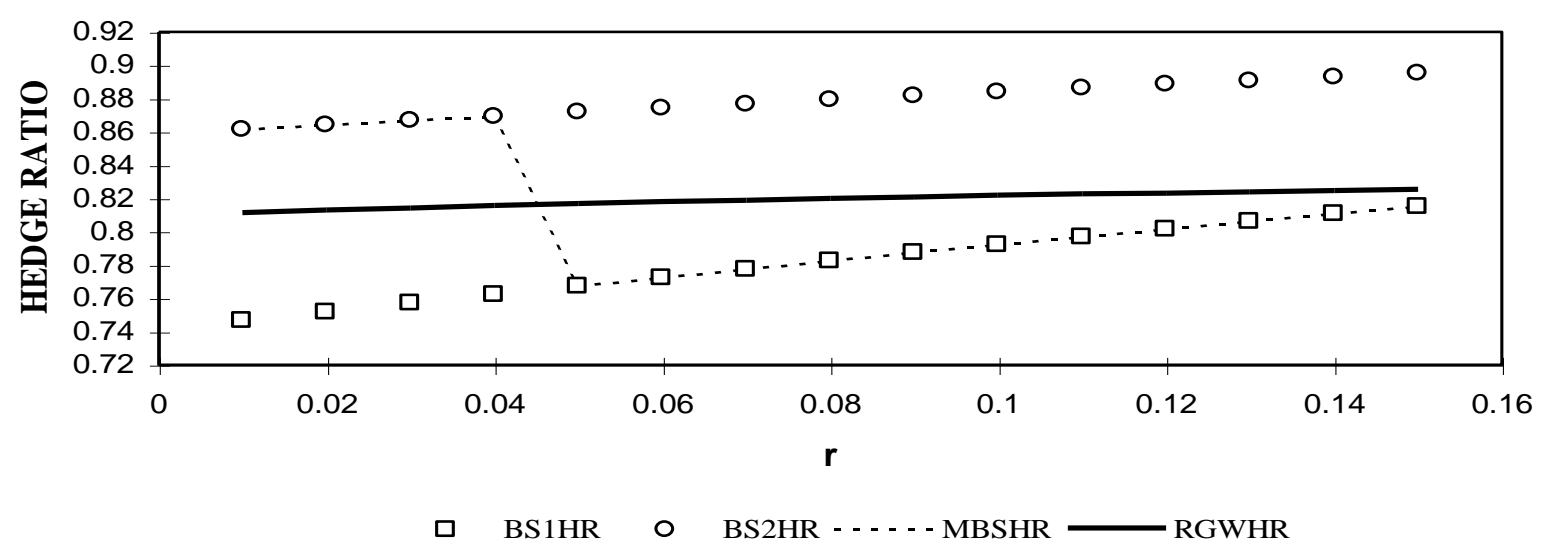

Figure 3B

\% DIFF HEDGE RATIOS

$\mathrm{P}=50 \mathrm{X}=45 \mathrm{~s}=\mathrm{s} \mathrm{t}=45 \mathrm{~T}=90 \mathrm{D}=1$

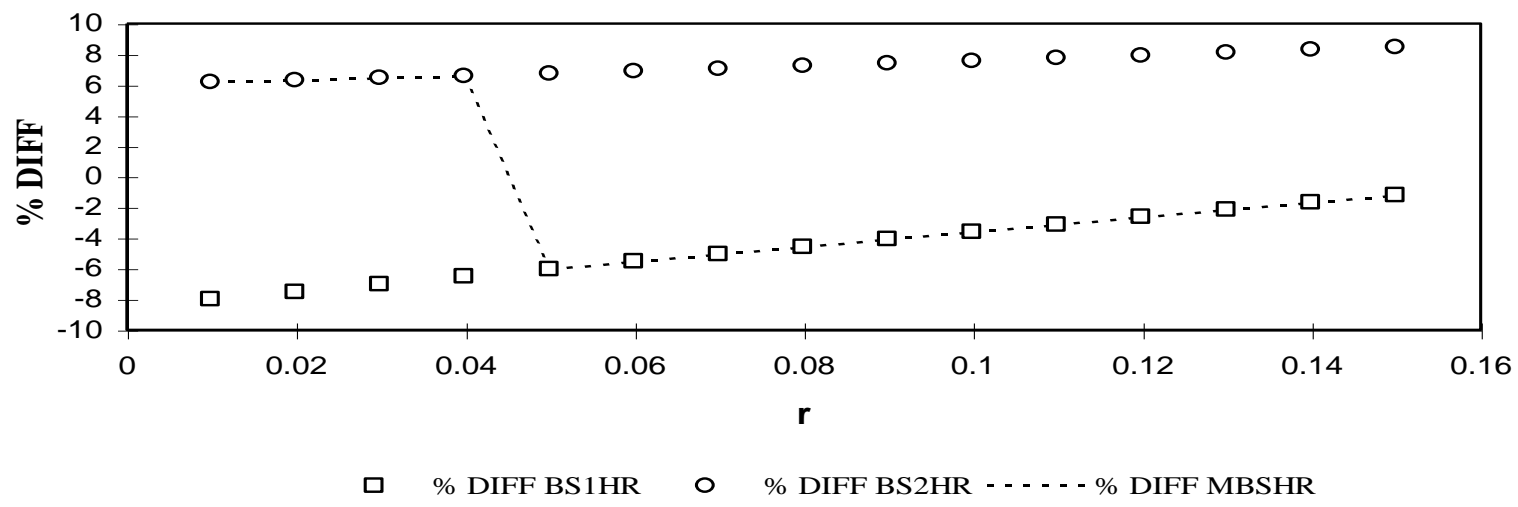

Figure 4. 


\section{Effect of Stock Volatility, $\sigma$, on Hedge Ratios}

Panel A shows the hedge ratios. Panel B shows the percentage difference between the modified Black-Scholes hedge ratios and the Roll-Geske-Whaley hedge ratios. BS1HR is the hedge ratio assuming no early exercise. BS2HR is the hedge ratio assuming early exercise. MBSHR is the hedge ratio associated with the larger Black-Scholes option value.

\section{Figure 4A}

\section{HEDGE RATIOS}

$P=55 \quad X=45 \mathrm{D}=1 \mathrm{t}=45 \mathrm{~T}=90 \mathrm{r}=.05$

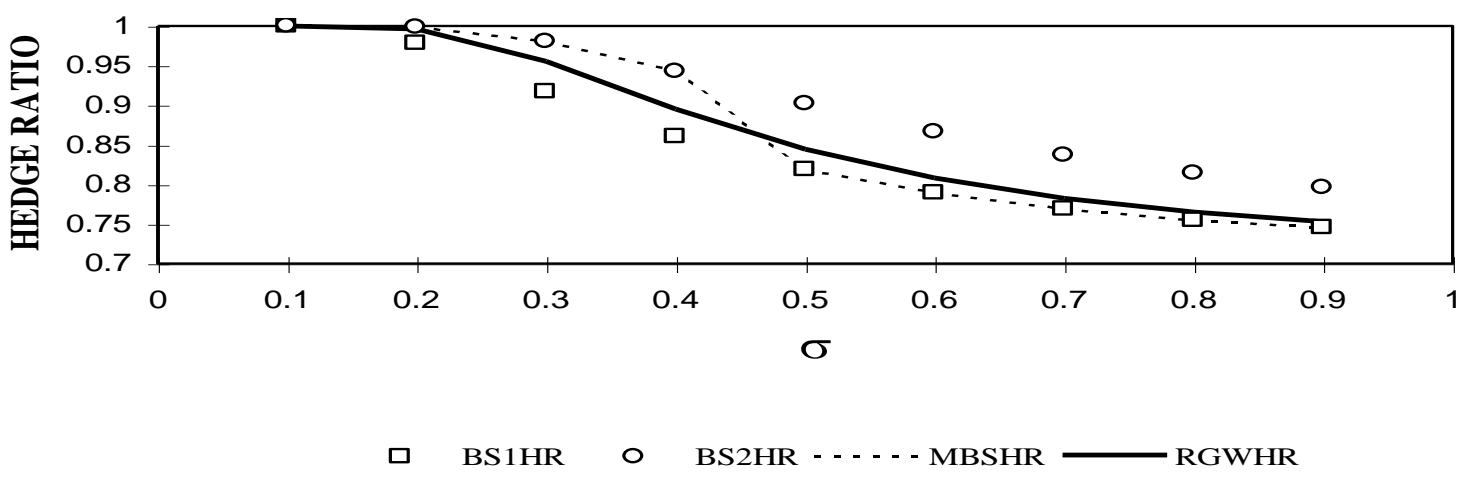

Figure 4B

\% DIFF HEDGE RATIOS

$\mathrm{P}=55 \mathrm{X}=45 \mathrm{D}=1 \mathrm{t}=45 \mathrm{~T}=90 \mathrm{r}=.05$

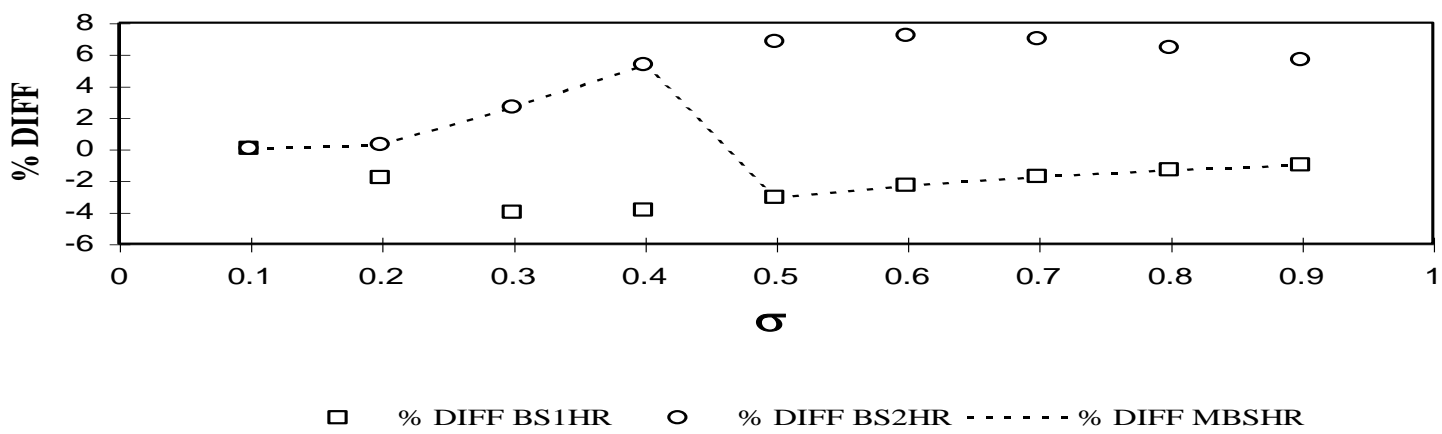

\section{Effect of Stock Volatility}

As the stock price standard deviation increases, the insurance benefit (described in Section I) associated with not exercising early increases. Thus, the likelihood of early exercise should decrease as the stock volatility increases, and the BS hedge ratio based on no early exercise, BS1HR, should be more accurate. The BS modification based on early exercise, BS2HR, should be more accurate for low values of volatility. This is verified in Figure 4.

As can be seen in Figure 4, the error associated with the modified BS approximation is zero at low levels of volatility and peaks out at about $5 \%$ at a volatility of $40 \%$. At a standard deviation of $50 \%$, the modified BS hedge ratio switches to the BS hedge ratio based on no early exercise and the BS1HR then becomes fairly accurate at high 
levels of volatility.

\section{Effect of Degree In-the-Money}

The probability of exercising the option at the ex-dividend date is small if the option is currently far out-ofthe-money. As the option becomes more in-the-money, the likelihood of exercising early increases. Therefore, the BS1HR hedge ratio should be a good approximation for relatively low stock prices, and BS2HR should be accurate for relatively large stock prices. In between, the approximation should be less accurate. This is verified in Figure 5.

As can be seen in Figure 5, the hedge ratios are zero and one for options that are extremely out-of or in-themoney respectively. The BS ratio assuming no early exercise is accurate for options that are extremely out-of-themoney. As the degree of in-the-money increases, the modified BS hedge ratio switches to the BS2HR at a stock price of $\$ 40$ and this approximation becomes exact for options which are extremely in-the-money. ${ }^{2}$ The absolute maximum error using the modified BS approximation is slightly more than $10 \%$ at a stock price of $\$ 35$.

\section{Summary and Conclusions}

The Black-Scholes option pricing model and its associated hedge ratio (modified to account for dividends on the underlying stock) have received much attention in the literature and in practice. Although the RGW model, which represents an improvement over the Black-Scholes model, has also received much attention, its associated hedge ratio has not. We derived an analytic formula for the hedge ratio based on the RGW option pricing model. We then sought to determine when and by how much the modified BS hedge ratios differ from the more accurate RGW-based hedge ratio.

It was argued that the difference between the more accurate RGW hedge ratio and the modified BS hedge ratio should be the greatest when the likelihood of early exercise is far away from zero or one. We discussed when this would occur. Important determinants are the size of the dividend, the degree to which the option is in or out-ofthe-money, the time to ex-dividend relative to the time remaining until expiration, the volatility of the return on the underlying stock, and the rate of interest. For certain values of these variables, the likelihood of early exercise will be small and the modified BS hedge ratio assuming no early exercise will be accurate. At other times the likelihood of early exercise will be large, and the modified BS approach assuming early exercise will give accurate hedge ratios. In between, the modified BS approach will not be accurate. Depending on the values of the important variables, the differences can be large.

\section{Endnotes}

1. At the $\$ 3.50$ dividend, the modified BS hedge ratio is equal to the BS1HR even though the BS2HR is more accurate. This is because the BS call value based on no early exercise is the larger of the two call values. The pseudo-American approach calls for selecting the larger call value and associated hedge ratio.

2. The BS approximation assuming no early exercise also eventually becomes exact, since all hedge ratios approach one for options which are extremely in-the-money.

Figure 5.

Effect of Stock Price, P, on Hedge Ratios 
Panel A shows the hedge ratios. Panel B shows the percentage difference between the modified Black-Scholes hedge ratios and the Roll-Geske-Whaley hedge ratios. BS1HR is the hedge ratio assuming no early exercise. BS2HR is the hedge ratio assuming early exercise. MBSHR is the hedge ratio associated with the larger Black-Scholes option value.

Figure 5A

\section{HEDGE RATIOS}

$X=45=.30 \mathrm{D}=1 \mathrm{t}=75 \mathrm{~T}=90 \mathrm{r}=.05$

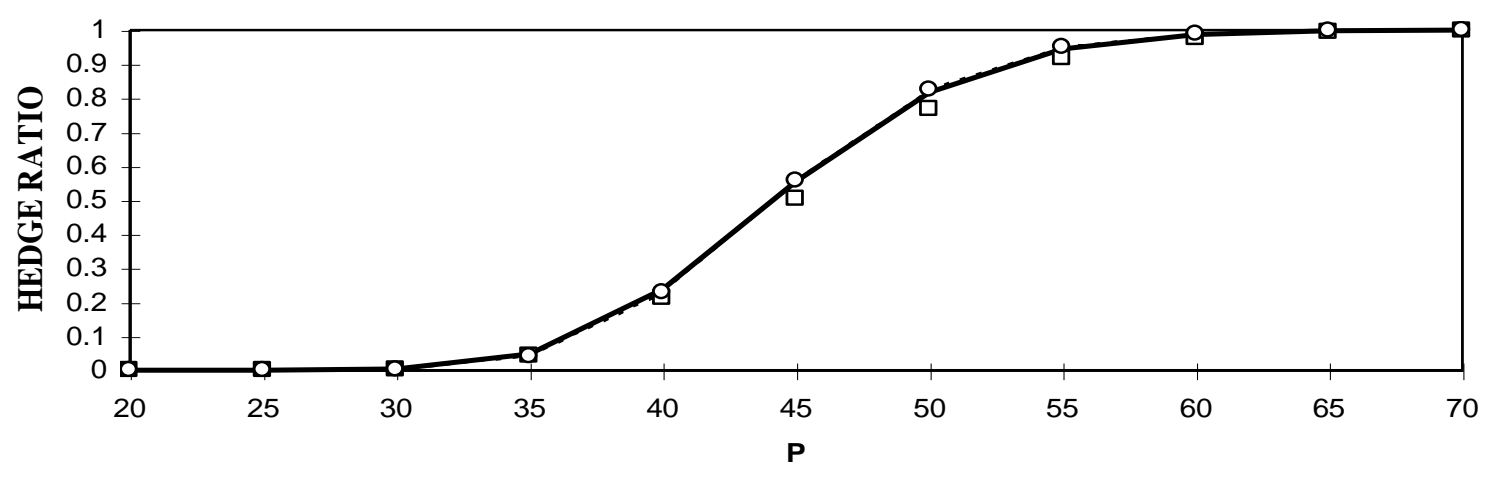

口 BS1HR O BS2HR - .... . MBSHR - RGWHR

Figure 5B

\% DIFF HEDGE RATIOS

$\mathrm{X}=45 \mathrm{D}=.30 \mathrm{D}=1 \mathrm{t}=75 \mathrm{~T}=90 \mathrm{r}=.05$

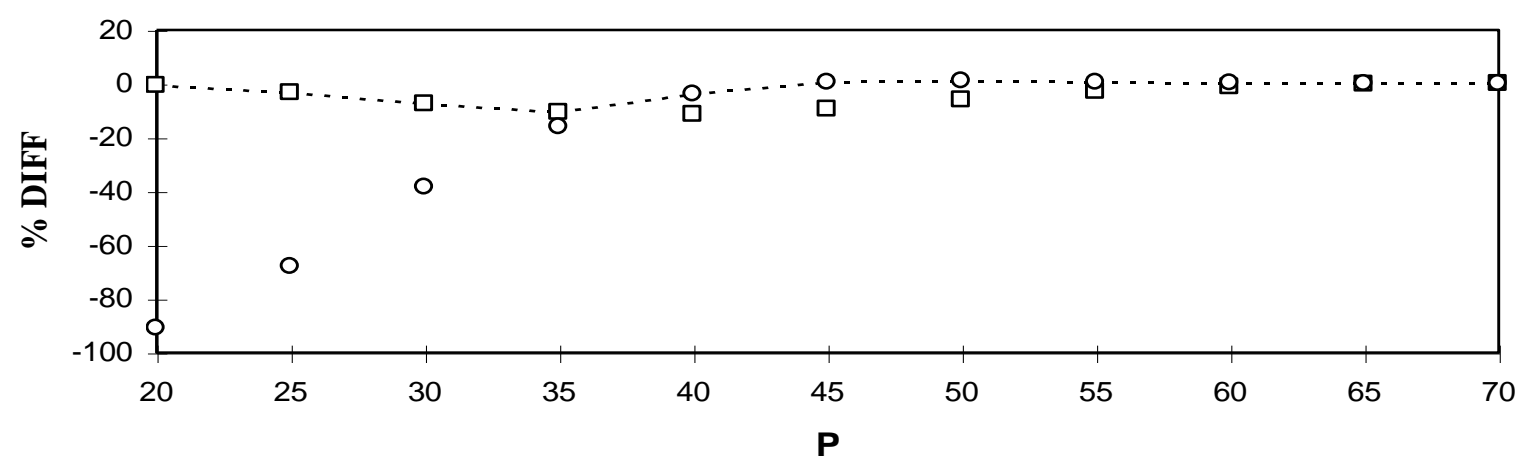

ㄷ DIFF BS1HR $\quad$ \% DIFF BS2HR - . . . \% DIFF MBSHR

\section{References}


1. Black, F., 1975, "Fact and Fantasy in the Use of Options," Financial Analysts Journal 31, 36-72.

2. Black, F., and M. Scholes, 1973, "The Pricing of Options and Corporate Liabilities," Journal of Political Economy 81, 637-659.

3. Cox, J., and M. Rubinstein, 1985, Options Markets, Englewood Cliffs, NJ: Prentice-Hall, 343-345.

4. Figlewski, S., Silber, W., and M. Subrahmanyam, 1992, Financial Options -- From Theory to Practice, Homewood Ill: Business One Irwin

5. Geske, R., 1979, "The Valuation of Compound Options,” Journal of Financial Economics 7, 63-81.

6. __ _ _ 1979, “A Note on Analytical Valuation Formula for Unprotected American Call Options on Stocks with Known Dividends," Journal of Financial Economics 7, 375-380.

7. Haugen, R., 1997, Modern Investment Theory, 4 th Ed., Englewood Cliffs, NJ: Prentice Hall

8. Hull, J., 2000, Options, Futures, and Other Derivative Securities, 4 th Ed., Englewood Cliffs, NJ: Prentice Hall

9. Jarrow R., and S. Turnbull, 2000, Derivative Securities, 2nd Ed., South-Western College Publishing

10. Ritchken, P., 1987, Options -- Theory, Strategy, and Applications, Glenview, Ill: Scott, Foresman and Company

11. Roll, R., 1977, “An Analytic Valuation Formula for Unprotected American Call Options on Stocks with Known Dividends," Journal of Financial Economics 5, 251-258.

12. Sharpe, W. F., G. J. Alexander and J. V. Bailey, 1999, Investments, 6th Ed., Englewood Cliffs, NJ: Prentice Hall

13. Sterk, W., 1983, "Comparative Performance of the Black-Scholes and Roll-Geske-Whaley Option Pricing Models," Journal of Financial and Quantitative Analysis 18, 345-354.

14. Whaley, R.W., 1981, "On the Valuation of American Call Options on Stocks with Known Dividends," Journal of Financial Economics 9, 207-211.

15. , 1982, "Valuation of American Call Options on Dividend-Paying Stocks -- Empirical Tests," Journal of Financial Economics 10, 29-58.

\section{Appendix A}

The RGW hedge ratio, RGWHR, is derived as follows:

Equation (1) from the paper can be rewritten as:

$$
R G W=S\left[N_{1}\left(b_{1}\right)+N_{2}\left(a_{1},-b_{1} ; \rho\right)\right]-P V(X, T)\left[N_{1}\left(b_{2}\right) A+N_{2}\left(a_{2},-b_{2} ; \rho\right)\right]+B N_{1}\left(b_{2}\right)
$$

Where

$$
\begin{aligned}
& a_{1}=\frac{\ln S-\ln [P V(X, T)]}{\sigma \sqrt{T}}+0.5 \sigma \sqrt{T} ; a_{2}=a_{1}-\sigma \sqrt{T} \\
& b_{1}=\frac{\ln S-\ln \left[P V\left(S_{t}^{*}, t\right)\right]}{\sigma \sqrt{t}}+0.5 \sigma \sqrt{t} ; b_{2}=b_{1}-\sigma \sqrt{t} \\
& P V(X, T)=X \exp (-r T) ; P V\left(S_{t}^{*}, t\right)=S_{t}^{*} \exp (-r t) ; \quad \rho=-\sqrt{\frac{t}{T}} \\
& N_{1}(a)=\int_{-\infty}^{a} f(z) d z ; f(z)=\frac{1}{\sqrt{2 \pi}} \exp \left(-0.5 z^{2}\right) \\
& N_{2}(a, b ; \rho)=\int_{-\infty-\infty}^{a} \int^{b} h(u, v ; \rho) d u d v
\end{aligned}
$$




$$
\begin{aligned}
& h(u, v ; \rho)=\frac{1}{2 \pi \sqrt{1-\rho^{2}}} \exp \left[\frac{-\left(u^{2}-2 \rho u v+v^{2}\right)}{2\left(1-\rho^{2}\right)}\right] \\
& A=\exp [r(T-t)] ; \quad B=\alpha D \exp (-r t) .
\end{aligned}
$$

The RGW hedge ratio is the partial derivative of the RGW call value with respect to $S$.

$$
\begin{aligned}
& R G W H R=\frac{\partial R G W}{\partial S}=N_{1}\left(b_{1}\right)+N_{2}\left(a_{1},-b_{1} ; \rho\right) \\
& +S\left[\frac{\partial N_{1}\left(b_{1}\right)}{\partial S}+\frac{\partial N_{2}\left(a_{1},-b_{1} ; \rho\right)}{S}\right]-P V(X, T)\left[\frac{\partial N_{1}\left(b_{2}\right)}{\partial S} A+\frac{\partial N_{2}\left(a_{2},-b_{2} ; \rho\right)}{\partial S}\right]+B \frac{\partial N_{1}\left(b_{2}\right)}{\partial S}
\end{aligned}
$$

To evaluate the above expression, use the following:

$$
\frac{\partial N_{1}(b)}{\partial S}=\frac{\partial N_{1}(b)}{\partial b} \frac{\partial b}{\partial S}=f(b) \frac{\partial b}{\partial S} \quad ; \quad b=b_{1}, b_{2}
$$

from the fundamental theorem of calculus which states that the derivative of a definite integral with respect to its upper limit is equal to the integrand evaluated at the upper limit.

$$
\begin{aligned}
& \frac{\partial N_{2}(a,-b ; \rho)}{\partial S}=\frac{\partial N_{2}(a,-b ; \rho)}{\partial a} \frac{\partial a}{\partial S}+\frac{\partial N_{2}(a,-b ; \rho)}{\partial(-b)} \frac{\partial(-b)}{\partial S} ; \\
& a=a_{1}, a_{2} ; b=b_{1}, b_{2}
\end{aligned}
$$

Where

$$
\begin{aligned}
& \frac{\partial N_{2}(a,-b ; \rho)}{\partial a}=\frac{\exp \left(-0.5 a^{2}\right)}{\sqrt{2 \pi}} N_{1}\left(\frac{-a \rho-b}{\sqrt{1-\rho^{2}}}\right) \\
& \frac{\partial N_{2}(a,-b ; \rho)}{\partial(-b)}=\frac{\exp \left(-0.5 b^{2}\right)}{\sqrt{2 \pi}} N_{1}\left(\frac{a+b \rho}{\sqrt{1-\rho^{2}}}\right) \\
& \frac{\partial a}{\partial S}=\frac{1}{S \sigma \sqrt{T}} ; \frac{\partial(-b)}{\partial S}=\frac{-1}{S \sigma \sqrt{T}} .
\end{aligned}
$$

The proof of (iii) and (iv) is as follows:

$$
\frac{\partial N_{2}(a, b ; \rho)}{\partial a}=\frac{\partial}{\partial a}\left[\int_{-\infty}^{a}\left[\int_{-\infty}^{b} h(u, v ; \rho) d u\right] d v\right]
$$

Where 


$$
h(u, v ; \rho)=\frac{1}{2 \pi \sqrt{1-\rho^{2}}} \exp \left[-0.5\left(\frac{u^{2}-2 \rho u v+v^{2}}{1-\rho^{2}}\right)\right]
$$

Again by the fundamental theorem of calculus

$$
\frac{\partial N_{2}}{\partial a}=\int_{-\infty}^{b} h(u, a ; \rho) d u=\int_{-\infty}^{b} \frac{1}{2 \pi \sqrt{1-\rho^{2}}} \exp \left[-0.5\left(\frac{u^{2}-2 \rho u a+a^{2}}{1-\rho^{2}}\right)\right] d u .
$$

Now

$$
(u-a \rho)^{2}=u^{2}-2 \rho u a+a^{2} \rho^{2}+a^{2}-a^{2}=u^{2}-2 \rho u a+a^{2}-a^{2}\left(1-\rho^{2}\right) .
$$

Therefore,

$$
\frac{(u-a \rho)^{2}}{\sqrt{1-\rho^{2}}}+a^{2}=\frac{u^{2}-2 \rho u a+a^{2}}{1-\rho^{2}} .
$$

Therefore,

$$
\begin{aligned}
& \frac{\partial N_{2}}{\partial a}=\int_{-\infty}^{b} \frac{1}{2 \pi \sqrt{1-\rho^{2}}} \exp \left[-0.5\left(\frac{u-a \rho}{\sqrt{1-\rho^{2}}}\right)^{2}\right] \exp \left[-0.5 a^{2}\right] d u \\
& =\frac{\exp \left[-0.5 a^{2}\right]}{\sqrt{2 \pi}} \int_{-\infty}^{b} \frac{1}{\sqrt{2 \pi} \sqrt{1-\rho^{2}}} \exp \left[-0.5\left(\frac{u-a \rho}{\sqrt{1-\rho^{2}}}\right)^{2}\right] d u .
\end{aligned}
$$

Let

$$
z=\frac{u-a \rho}{\sqrt{1-\rho^{2}}} \quad ; \quad d z=\frac{d u}{\sqrt{1-\rho^{2}}} .
$$

Changing variables from $u$ to $z$,

$$
\frac{\partial N_{2}}{\partial a}=\frac{\exp \left[-0.5 a^{2}\right]}{\sqrt{2 \pi}} \int_{-\infty}^{\frac{b-a \rho}{\sqrt{1-\rho^{2}}}} \frac{1}{\sqrt{2 \pi}} \exp \left[-0.5 z^{2}\right] d z=\frac{\exp \left[-0.5 a^{2}\right]}{\sqrt{2 \pi}} N_{1}\left(\frac{b-a \rho}{\sqrt{1-\rho^{2}}}\right) .
$$

The expression $\mathrm{N}_{2}(a, b ; \rho)$ is exactly symmetrical in $a$ and $b$, hence

$$
\frac{\partial N_{2}}{\partial b}=\frac{\exp \left[-0.5 b^{2}\right]}{\sqrt{2 \pi}} N_{1}\left(\frac{a-b \rho}{\sqrt{1-\rho^{2}}}\right) .
$$

Finally, to get the required results, substitute $-b$ for $b$. 
Substituting equations $i$ through $v$ into equation A-2 gives

$$
\begin{aligned}
& R G W H R=N_{1}\left(b_{1}\right)+N_{2}\left(a_{1},-b_{1} ; \rho\right) \\
& +S\left\{\frac{1}{\sqrt{2 \pi}} \exp \left[-0.5 b_{1}^{2}\right]\left(\frac{1}{S \sigma \sqrt{t}}\right)+\frac{\exp \left[-0.5 a_{1}^{2}\right]}{\sqrt{2 \pi}} N_{1}\left(\frac{-a_{1} \rho-b_{1}}{\sqrt{1-\rho^{2}}}\right) \frac{1}{S \sigma \sqrt{T}}\right. \\
& \left.+\frac{\exp \left[-0.5 b_{1}^{2}\right]}{\sqrt{2 \pi}} N_{1}\left(\frac{a_{1}+b_{1} \rho}{\sqrt{1-\rho^{2}}}\right)\left(\frac{-1}{S \sigma \sqrt{t}}\right)\right\} \\
& -X \exp [-r T]\left\{\frac{1}{\sqrt{2 \pi}} \exp \left[-0.5 b_{2}^{2}\right]\left(\frac{\exp [r(T-t)]}{S \sigma \sqrt{t}}\right)+\frac{\exp \left[-0.5 a_{2}^{2}\right]}{\sqrt{2 \pi}} N_{1}\left(\frac{-a_{2} \rho-b_{2}}{\sqrt{1-\rho^{2}}}\right) \frac{1}{S \sigma \sqrt{T}}\right. \\
& \left.+\frac{\exp \left[-0.5 b_{2}^{2}\right]}{\sqrt{2 \pi}} N_{1}\left(\frac{a_{2}+b_{2} \rho}{\sqrt{1-\rho^{2}}}\right)\left(\frac{-1}{S \sigma \sqrt{t}}\right)\right\} \\
& +\alpha D \exp [-r t]\left(\frac{1}{\sqrt{2 \pi}} \exp \left[-0.5 b_{2}^{2}\right]\right) \frac{1}{S \sigma \sqrt{t}} .
\end{aligned}
$$

This simplifies somewhat to equation A-3.

$$
\begin{aligned}
& R G W H R=N_{1}\left(b_{1}\right)+N_{2}\left(a_{1},-b_{1} ; \rho\right)+\frac{1}{S \sqrt{2 \pi} \sigma}\{ \\
& S\left[\frac{\exp \left[-0.5 b_{1}^{2}\right]}{\sqrt{t}}+\frac{\exp \left[-0.5 a_{1}^{2}\right]}{\sqrt{T}} N_{1}\left(\frac{-a_{1} \rho-b_{1}}{\sqrt{1-\rho^{2}}}\right)-\frac{\exp \left[-0.5 b_{1}^{2}\right]}{\sqrt{t}} N_{1}\left(\frac{a_{1}+b_{1} \rho}{\sqrt{1-\rho^{2}}}\right)\right] \\
& -X \exp [-r T]\left[\frac{\exp \left[-0.5 b_{2}^{2}\right]}{\sqrt{t}} \exp [r(T-t)]+\frac{\exp \left[-0.5 a_{2}^{2}\right]}{\sqrt{T}} N_{1}\left(\frac{-a_{2} \rho-b_{2}}{\sqrt{1-\rho^{2}}}\right)\right. \\
& \left.\left.-\frac{\exp \left[-0.5 b_{2}^{2}\right]}{\sqrt{t}} N_{1}\left(\frac{a_{2}+b_{2} \rho}{\sqrt{1-\rho^{2}}}\right)\right]+\frac{\alpha D \exp [-r t]}{\sqrt{t}} \exp \left[-0.5 b_{2}^{2}\right]\right\} .
\end{aligned}
$$

\section{Notes}

